\title{
Efeito de previsibilidade temporal de perturbações posturais e demanda de precisão de tarefa manual no desempenho em tarefa dual
}

http://dx.doi.org/10.11606/1807-5509202000020295

\author{
Aline Grandi da SILVA* \\ Daniel Boari COELHO \\ Catarina Costa BOFFINO**** \\ Luis Augusto TEIXEIRA** \\ Andrea Cristina de LIMA-PARDINI ${ }^{* * *}$
}

*Departamento de

Fisioterapia, Fonoaudiologia e Terapia Ocupacional, Faculdade de Medicina, Universidade de São Paulo, São Paulo, SP, Brasil.

${ }^{* *}$ Escola de Educação Física e Esporte, Universidade de São Paulo, São Paulo, SP, Brasil.

***Instituto de Radiologia, Hospital das Clínicas, Faculdade de Medicina, Universidade de São Paulo, São Paulo, SP, Brasil.

\section{Introdução}

Por um longo tempo, o controle postural foi considerado apenas como uma resposta automática, controlado por circuitos medulares, aos estímulos do ambiente. Estudos recentes, no entanto, têm mostrado que o controle postural envolve um complexo e ativo sistema de controle mediado por estruturas superiores corticais ${ }^{1}$. Evidências têm sugerido uma interação entre níveis de controle de movimento sob regulação cortical (por exemplo: exigência de precisão de movimentos voluntários) e níveis de controle de movimento sob regulação subcortical (controle do equilíbrio postural). Estudos têm mostrado que, durante tarefas manuais que exigem precisão motora, como em movimentos oculares finos para a percepção visual ${ }^{2,3}$ ou movimentos manuais ${ }^{4,5}$, há uma diminuição da oscilação postural. MorioKa, HiYAmizU e YAGI ${ }^{5}$ avaliaram o comportamento postural contrastando uma tarefa de segurar um copo cheio de água contra um copo vazio. Os resultados indicaram que ao segurar um copo cheio de água obteve-se diminuição da oscilação corporal. Dado que maior estabilidade corporal é um pré-requisito para a manutenção estável do copo na mão, é plausível que o desempenho na tarefa manual também seja beneficiado. Resultados análogos foram observados quando movimentos oculares finos eram realizados para a busca visual ${ }^{6}$, a mudança do foco visual para acompanhar a oscilação horizontal de um alvo visual ${ }^{7}$ e de detecção de sinais visuais ${ }^{8}$. Supostamente, nestas situações menor oscilação do corpo na postura ereta favoreceu os movimentos mais precisos do sistema cabeça-olhos para o desempenho das tarefas visuais. Estes resultados 
foram interpretados como consequência da integração funcional do controle postural com a tarefa manual ${ }^{7,8}$, como originalmente proposto por STOFFregen e Riccio9 9 . Desta forma, o controle postural envolve mais funçóes do que somente minimizar a oscilação corporal. A coordenação motora apropriada dentro de um ambiente que se altera constantemente pode exigir movimentos posturais modulados com base nas restriçôes colocadas por uma tarefa manual ${ }^{10}$.

A adaptação do controle postural de acordo com o ambiente tem sido observada também em situações em que o indivíduo é perturbado externamente. Tem sido verificado que quando o indivíduo tem conhecimento das características da perturbação postural (por exemplo, momento ou magnitude da perturbação) as respostas reativas são mais bem adaptadas ${ }^{11-16}$. HoraK, Diener e Nashner ${ }^{11}$ demonstraram que a magnitude de respostas posturais foi relacionada à expectativa gerada por pistas sobre a amplitude do deslocamento da superfície de apoio. Desta forma, quando o indivíduo esperava uma perturbação de alta amplitude suas respostas posturais, caracterizada pela amplitude de deslocamento do centro de pressão, eram maiores do que quando era esperada uma perturbação de baixa amplitude. Outra evidência para esta adaptação do controle postural foi fornecida através de medições neurofisiológicas ${ }^{17-20}$, demonstrando o aumento da ativação da área motora suplementar e das áreas sensório-motoras primárias na situação de previsibilidade da perturbação postural. Esta maior ativação foi correlacionada com a menor oscilação postural do sujeito.

De Lima, Azevedo Neto e Teixeira ${ }^{12}$ verificaram que, além da previsibilidade da perturbação postural, a maior demanda de exigência de estabilidade da tarefa manual levaram a respostas posturais mais adaptadas. Neste estudo, os participantes deveriam recuperar a estabilidade após perturbaçóes do equilíbrio corporal por meio de translação da plataforma de apoio, comparando-se perturbações com direção previsível e imprevisível. Para testar o efeito da restrição da tarefa manual, os participantes deveriam segurar uma bandeja com ou sem um cilindro sobre ela (alta e baixa restrição, respectivamente). Os resultados mostraram que na condição previsível com alta restrição houve diminuição da latência de ativação dos músculos agonistas e do deslocamento angular das principais articulações envolvidas com a manutenção do controle postural. Tais resultados indicam que o conhecimento do momento da perturbação associado à maior demanda de controle manual levou a ajustes posturais mais efetivos. No entanto, a análise do desempenho da tarefa manual foi insuficiente para levar a uma maior compreensão da integração entre o controle postural e manual. Os autores calcularam o deslocamento horizontal da bandeja, mas não correlacionaram esta variável com o desempenho postural, o que poderia levar a interpretações mais robustas sobre a suposta integração entre elas de acordo com o contexto.

O objetivo deste estudo foi avaliar a integração entre os componentes manual e postural em uma tarefa motora dual em resposta a perturbações que afetam a ambos os componentes. Esta avaliação foi feita em função da demanda de precisão da tarefa manual (projeção do laser em alvos de diferentes tamanhos) e da previsibilidade do momento da perturbação (por meio de dicas temporais). Considerando que tanto a previsibilidade da perturbação $^{11,12}$ quanto à demanda de precisão manual ${ }^{9,10}$ podem modular respostas posturais reativas, nós hipotetizamos que a condição de perturbação postural temporalmente previsível associada à alta demanda de precisão da tarefa manual induz menor oscilação corporal (maior estabilidade postural) e menor deslocamento da projeção do laser no alvo (maior estabilidade manual), afetando a integração entre as tarefas postural e manual.

\section{Método}

\section{Participantes}

Participaram do estudo oito voluntários adultos jovens com idade entre 20-30 anos $(\mathrm{M}=23,63$ anos, $\mathrm{DP}=2,56)$, com altura média de $1,69 \mathrm{~m}(\mathrm{DP}=0,07)$, sendo 5 mulheres.

Os participantes declararam não apresentar qualquer alteração neuromuscular, sensorial ou cognitiva que comprometesse o equilíbrio, além de não utilizarem medicamentos que pudessem comprometer a atenção ou o equilíbrio. No caso de alterações visuais, as mesmas foram corrigidas. 
Os participantes foram recrutados na Escola de Educação Física e Esporte e no Centro de Práticas Esportivas da Universidade de São Paulo e assinaram o termo de consentimento livre e esclarecido sobre a pesquisa para ingressar no estudo, o qual foi aprovado pelo comitê de ética local.

\section{Tarefas e Equipamentos}

A tarefa experimental foi de caráter dual, consistindo em manter a estabilidade da postura ereta e simultaneamente manter a pontaria para um alvo espacial durante as respostas a uma perturbação mecânica. A postura inicial consistia em manter-se em pé sobre uma plataforma de força (EMG System), segurando com as duas mãos uma estrutura de madeira $(30 \mathrm{~cm} \times 15 \mathrm{~cm}$ x $20 \mathrm{~cm}) \mathrm{com}$ uma ponteira laser fixada em sua face superior. $\mathrm{O}$ componente manual da tarefa consistia em fazer pontaria para um alvo vertical disposto a uma distância de $2 \mathrm{~m}$ do participante, aproximadamente à altura de seus ombros. Os dois braços deveriam ser mantidos em paralelo com o solo, à altura dos ombros, com cotovelos estendidos. $\mathrm{Na}$ face anterior da estrutura de madeira havia um sistema eletromagnético para carregamento e liberação de carga. Em todas as condições avaliadas, uma carga de $2,5 \mathrm{Kg}$ era conectada ao sistema por meio de um cabo de aço, aplicando uma força de tração no sentido anterior (FIGURA 1). A carga era liberada remotamente pelo experimentador, fazendo com que houvesse uma oscilação corporal posterior, perturbando simultaneamente a pontaria ao alvo e a estabilidade da postura ereta.

O deslocamento da ponteira laser foi rastreado por meio de marcadores colocados nas porções anterior e posterior do dispositivo de emissão do laser. Estes marcadores foram rastreados por sistema composto por quatro câmeras optoeletrônicas (Vicon, Nexus). A sincronização dos sinais das câmeras e da plataforma de força, tendo como marco temporal o sinal de liberação de carga, foi feito no sistema Vicon.

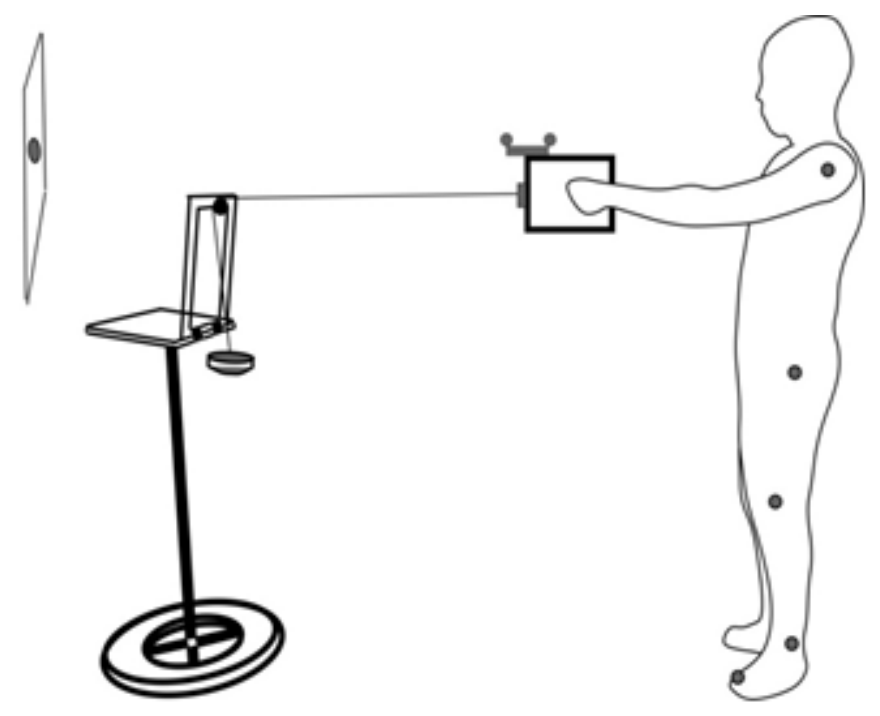

FIGURA 1 -Representação esquemática do arranjo experimental, mostrando a posição inicial do indivíduo (segurando a estrutura de suporte do laser com as duas mãos), o dispositivo de liberação de carga e os marcadores cinemáticos nas articulações e na ponteira de emissão de laser.

\section{Delineamento experimental e procedimentos}

Os participantes eram inicialmente instruídos a respeito do duplo objetivo da tarefa motora: (a) para o componente manual, manter a projeção do laser dentro dos limites do alvo; e (b) para o componente postural, manter o equilíbrio da postura ereta sem tirar os pés do lugar. Ambos os componentes eram igualmente enfatizados na instrução, de forma a não haver priorização de algum componente. Esta instrução se aplicava tanto à época pré-perturbação quanto pós-perturbação. Os participantes eram instruídos também a evitar açōes antecipatórias à perturbação. Durante a avaliação, os participantes eram testados em quatro condiçõos experimentais resultantes da combinação de dois fatores: (a) 
previsibilidade sobre o momento da perturbação e (b) demanda de precisão da tarefa manual. Para manipular previsibilidade temporal, foram comparadas duas condiçôes: liberação de carga em tempo previsível versus imprevisível. $\mathrm{Na}$ condição de imprevisibilidade temporal da perturbação (IP) a carga era liberada em tempos variados aleatoriamente dentro de uma janela temporal de $5 \mathrm{~s}$, de forma imprevisível para o participante. $\mathrm{Na}$ condição de certeza temporal da perturbação $(\mathrm{CP})$, a carga era liberada em um intervalo constante de 2 $s$ após um sinal verbal dado pelo experimentador. $\mathrm{O}$ participante era instruído sobre esta característica. O efeito da demanda de precisão (restrição) da tarefa manual foi avaliado por meio do tamanho do alvo circular no qual a projeção do laser deveria ser mantida. A condição de alta restrição (AR) foi realizada com um alvo circular de $2 \mathrm{~cm}$ de diâmetro, enquanto que na condição de baixa restrição (BR) o alvo circular media $20 \mathrm{~cm}$ de diâmetro.

Nos casos em que o participante tirava os pés do local inicial, a tentativa era cancelada e imediatamente repetida. Eram realizadas 5 repetições em cada condição experimental. No bloco de imprevisibilidade temporal da perturbação foram adicionadas 2 tentativas sem perturbação (uma para a condição de AR e uma para a condição de BR) para prevenir ajustes posturais antecipatórios. Desta forma, cada participante realizava ao todo 20 tentativas válidas para análise. Entre as tentativas era oferecido um período de descanso de $30 \mathrm{~s}$, intervalo em que a estrutura contendo a ponteira laser apoiada em um suporte para liberar e relaxar os braços do participante. Após cinco repetições em cada condição experimental eram dados 2 minutos de descanso. A ordem dos blocos das condições experimentais foi contrabalanceada entre os participantes.

\section{Análise}

As seguintes variáveis foram extraídas por meio do software Matlab (MathWorks):

Desempenho da tarefa manual: (A) Amplitude de deslocamento do laser: foi verificada a amplitude máxima de deslocamento do laser na direção vertical logo após a perturbação, em relação ao o valor da posição do laser no momento da perturbação. Tal variável indica a precisão de pontaria do laser no alvo; quanto menor o valor, mais precisa a pontaria. (B)

Velocidade máxima de deslocamento vertical do laser: dada pela velocidade máxima posterior à perturbação, subtraindo-se o valor da velocidade no momento da perturbação.

Desempenho no controle postural: Amplitude de deslocamento do centro de pressão nas direçôes (A) anteroposterior (CPap) e (B) mediolateral (CPml): foi analisada a amplitude máxima que o $\mathrm{CP}$ atingiu no sentido anteroposterior $\left(\mathrm{CPap}_{\max }\right)$ e mediolateral $\left.(\mathrm{CPml})_{\max }\right)$ imediatamente após a perturbação. $\mathrm{O}$ cálculo foi feito por meio da subtração do valor do CPap no momento da perturbação do valor máximo do CP. Quanto menor o valor, maior a estabilidade postural.

Integração entre os componentes postural e manual: Correlação cruzada do deslocamento do laser e do CPap, e entre o deslocamento do laser e do CPml. Esta análise indica a correlação interindividual para cada tentativa entre os sinais respectivos às duas variáveis ao longo do tempo e a defasagem temporal entre ambas no momento de maior correlação. As análises de defasagem temporal foram feitas em relação ao $\mathrm{CP}$, de forma que valores positivos indicam que o laser está atrasado em referência ao $\mathrm{CP}$.

A análise das variáveis foi feita por meio de ANOVA de dois fatores, 2 (previsibilidade) x 2 (restrição) para medidas repetidas em ambos os fatores. As comparaçôes post hoc foram feitas através de procedimentos de Newman-Keuls, com nível de significância de 5\%. As análises foram feitas através do programa Statistica (Statsoft). Serão relatados os efeitos significantes apenas.

\section{Resultados}

A fim de representar o efeito gerado pela liberação de carga nas diferentes variáveis analisadas, são mostrados na FIGURA 2 os sinais de tentativas individuais representativas de (a) deslocamento e (b) velocidade do laser, deslocamento de (c) CPap e de (d) CPml. O momento de liberação da carga é representado pelas linhas verticais tracejadas. Nesta figura são representadas as variações abruptas nas variáveis analisadas imediatamente após a liberação de carga. 


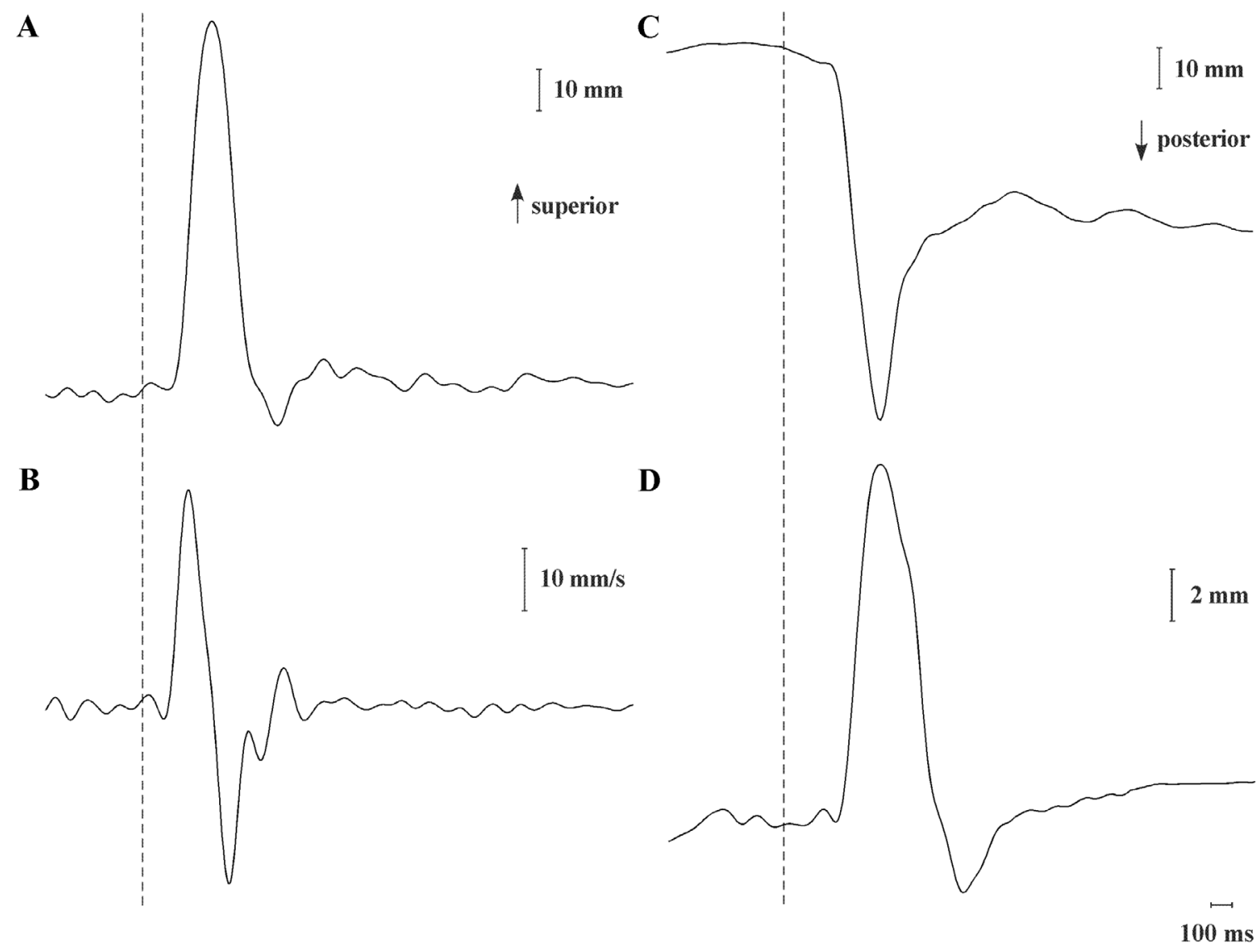

FIGURA 2 -Representação das curvas de deslocamento (A) e velocidade (B) do laser, do deslocamento de (C) CPap e (D) CPml. A linha vertical indica o momento de liberação da carga. As setas representam a direção de deslocamento.

\section{Centro de pressão}

A análise da amplitude CPap apontou efeito principal significante para previsibilidade, $F(1$, 7) $=13,53, p<0,001$, e interação significante entre previsibilidade e restrição, $\mathrm{F}(1,7)=6,32, \mathrm{p}=0,04$. Nas condições de perturbação previsível os valores de deslocamento do laser foram inferiores $(M=85,97$ $\mathrm{mm}, \mathrm{EP}=4,43)$ comparando-se às condiçóes de imprevisibilidade $(\mathrm{M}=95,62 \mathrm{~mm}, \mathrm{EP}=3,65)$. A interação foi devida ao fato de que na condição previsível com baixa restrição os valores de amplitude do CPap foram inferiores comparados às demais condiçôes (FIGURA 3A). A análise da amplitude do $\mathrm{CPml}$ indicou interação significante entre previsibilidade e restrição, $\mathrm{F}(1,7)=9,57, \mathrm{p}=$ 0,02 , o que foi devido a menores valores na condição previsível com baixa restrição em relação às demais condiçôes (FIGURA 3B).

\section{Tarefa manual}

A análise da amplitude de deslocamento do laser apontou efeito principal significante para previsibilidade, $F(1,7)=10,85$, $p=0,01$, com valores inferiores na condição previsível $(M=145,63 \mathrm{~mm}$, $\mathrm{EP}=12,10)$ em comparação à condição imprevisível $(\mathrm{M}=196,24 \mathrm{~mm}, \mathrm{EP}=11,18)$. A análise da velocidade máxima de deslocamento do laser apontou efeito principal significante para previsibilidade $\mathrm{F}(1$, 7) $=16,61, p<0,001$. Na condiçáo de previsibilidade encontraram-se valores inferiores $(M=73,33$ $\mathrm{mm} / \mathrm{s}, \mathrm{EP}=6,83)$ em comparação à condição de imprevisibilidade $(\mathrm{M}=102,58 \mathrm{~mm} / \mathrm{s}, \mathrm{EP}=8,31)$ (FIGURA 3C).

A análise de correlação cruzada entre CPap e deslocamento do laser indicou valores elevados, com média geral de $0,90(\mathrm{EP}=0,02)$ e defasagem temporal média de 36,47 ms ( $\mathrm{EP}=6,62)$.

A comparação dos valores de correlação e de defasagem temporal entre as condiçóes experimentais por meio de ANOVA indicou ausência de efeitos significantes em ambas as variáveis.

A análise da integração entre CPml e deslocamento do laser no eixo médio-lateral indicou que a média geral de correlação foi de $0,89(\mathrm{EP}=0,02)$, enquanto 
que a média geral de defasagem foi de $28,61 \mathrm{~ms}$ $(\mathrm{EP}=9,34)$. A ANOVA apontou efeito principal significante de previsibilidade, $\mathrm{F}(1,7)=6,59$, $\mathrm{p}=0,04$. $\mathrm{Na}$ condição previsível $(\mathrm{M}=0,85, \mathrm{EP}=0,04)$ a correlação foi menor em comparação à condição imprevisível $(\mathrm{M}=0,93, \mathrm{EP}=0,02)$. A análise de defasagem não apontou diferenças significantes entre as condiçóes (FIGURA 3D).
A
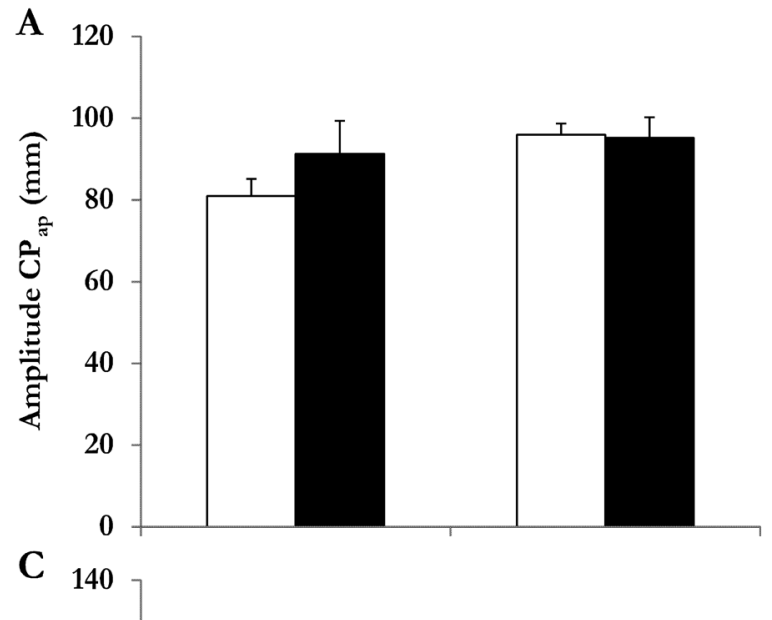

B
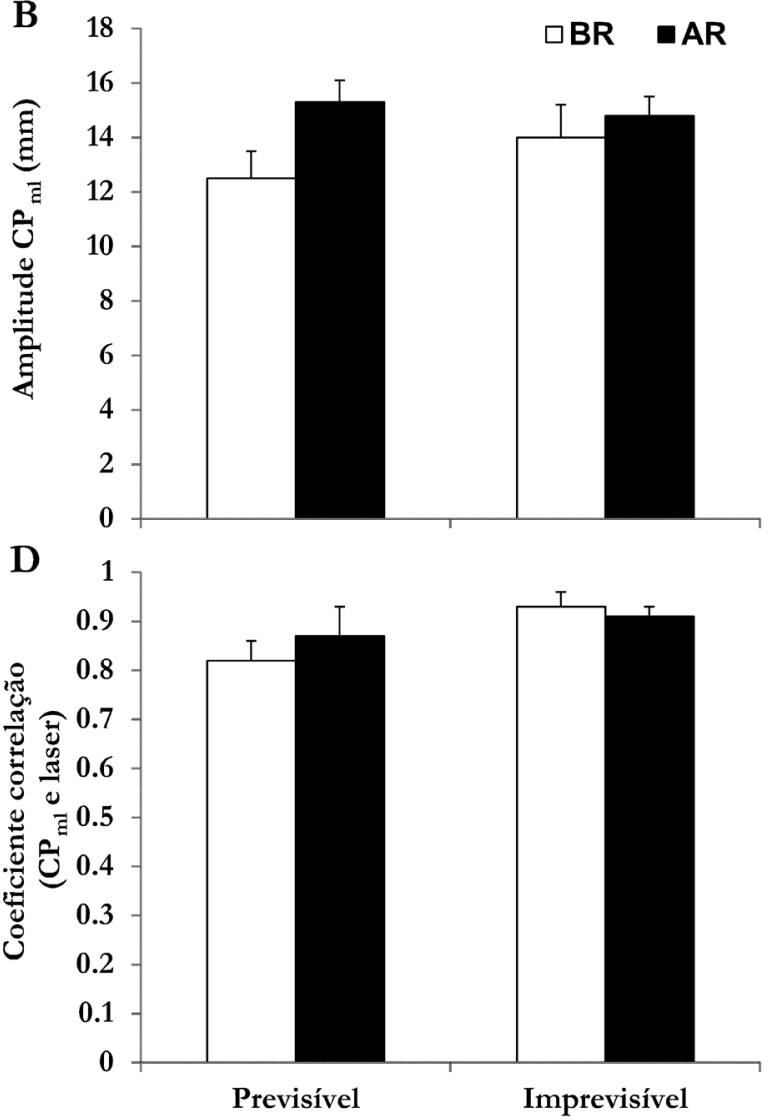

FIGURA 3 -Comparação de médias (erro padrão representado por barras verticais) para a amplitude do CP na direção anteroposterior (A) e mediolateral (B), amplitude de velocidade do laser (C) e coeficiente de correlação entre os deslocamentos de CP na direção mediolateral e do deslocamento vertical do laser (D) em função da condição de restrição e previsibilidade.

\section{Discussão}

O presente estudo teve como objetivo avaliar a integração entre os componentes manual e postural em uma tarefa motora dual em resposta a perturbações que afetam a ambos os componentes. Os resultados mostraram que a previsibilidade temporal da perturbação postural modulou o desempenho tanto na tarefa postural quanto na tarefa manual. Nas condiçôes de previsibilidade temporal, o deslocamento e velocidade da projeção do laser foram menores em comparação à condição de perturbação temporalmente imprevisível.
Diferentemente do que foi hipotetizado, os participantes não tiveram o desempenho na tarefa manual afetado pela restrição espacial imposta pelo tamanho do alvo. Contrariamente à nossa hipótese, a baixa demanda de precisão espacial na tarefa manual associada à previsibilidade temporal levou à menor oscilação corporal. Surpreendentemente, em condiçôes imprevisíveis a integração entre tarefa manual e controle postural foi maior do que em condições previsíveis.

O fato de o desempenho na tarefa manual 
náo ter sido afetado pela demanda de precisão na tarefa de pontaria poderia ser explicado pela perturbação aplicada na própria estrutura suportando a ponteira laser. Este método diferiu de estudos prévios mostrando o efeito da demanda de estabilidade da tarefa manual sobre respostas posturais automáticas, em que a perturbação foi aplicada ao tronco do participante ${ }^{13}$ ou por meio de deslocamento de sua base de suporte ${ }^{12,14}$. A perturbaçáo aplicada diretamente nos membros superiores pode ter levado a deslocamentos com magnitudes exageradas dos braços, possivelmente obscurecendo ajustes finos de posicionamento que poderiam ter sido eliciados de acordo com a demanda de precisão colocada pela tarefa manual. Apesar da possível dificuldade em realizar ajustes finos de acordo com a restriçáo espacial da tarefa manual, observou-se que o desempenho na tarefa manual foi melhor nas condiçóes temporalmente previsíveis em comparação às condiçôes em que o indivíduo não sabia o momento da perturbação. Em concordância a estes achados, nossos resultados evidenciaram que em condiçóes de previsibilidade houve diminuição da oscilação postural em situaçóes previsíveis. Evidências apontam que a informação sobre o momento da perturbação é capaz de modular as respostas reativas posturais, otimizando-as ${ }^{21-25}$. Tal otimização das respostas posturais reativas de acordo com a previsibilidade da perturbação postural envolve a participação de estruturas corticais como o córtex parietal posterior e área motora suplementar ${ }^{17,19,26,27}$.

Enquanto o córtex parietal posterior estaria envolvido na modulação das respostas posturais devido ao processamento de informações sensoriais multimodais relevantes ${ }^{28}$, detecção de instabilidade postural ${ }^{29}$ e representação dinâmica do esquema $\operatorname{corporal}^{30}$, a área motora suplementar utilizaria as informações previsíveis na seleção de programas motores adequados ao contexto ${ }^{18,31-33}$. Verifica-se, portanto, que o sistema nervoso central utiliza as informaçôes sobre o momento da perturbação postural, modulando tanto as respostas manuais quanto as posturais reativas.

De particular interesse para o presente estudo, foi encontrado que a oscilação corporal foi reduzida somente na condição de previsibilidade temporal em associação com baixa restrição manual. Estudos recentes evidenciam que em condiçôes nas quais o objetivo da tarefa manual exige maior estabilidade a magnitude de oscilação postural diminuii ${ }^{2-5,16,34}$. A divergência de nossos resultados em relação a estes achados prévios pode ser devida à elevada demanda atencional requisitada pela exigência de precisão da tarefa de alta restrição manual ${ }^{35-38}$. Essa característica pode ter levado a uma baixa priorização da tarefa de equilíbrio corporal, e como consequência a respostas posturais automáticas menos efetivas. Estudos recentes suportam esta perspectiva ao mostrarem que a requisição de recursos atencionais pela realização simultânea de tarefa cognitiva leva a oscilações corporais de maior amplitude em resposta a perturbações do equilíbrio corporal ${ }^{16,39,40}$. Desta forma, nas condiçôes de alta restrição e imprevisibilidade, mais recursos atencionais podem ter sido alocados para a realização das duas tarefas, prejudicando a efetividade das respostas posturais à perturbação. Por outro lado, na condição de baixa restrição, em que supostamente há menor alocação atencional para a tarefa manual ${ }^{35-38}$, a estabilidade postural pode ter sido beneficiada tanto pela maior disponibilidade de recursos atencionais quanto pela priorização das respostas posturais.

A integração entre os componentes postural (eixo médio-lateral) e manual da tarefa dual mostrou ser maior nas condiçōes imprevisíveis do que nas condições previsíveis. A menor integração nas condições previsíveis pode ser resultado de maior dissociação entre os movimentos manuais e posturais no intuito de atingir os objetivos dos dois componentes da tarefa dual ${ }^{41}$. Tal fato leva à suposição de que o controle postural reativo e a tarefa manual são controlados por sistemas integrados, porém independentes ${ }^{4,42,43}$. Apesar da alta correlação em todas as condições, quando o momento da perturbação era conhecido havia o favorecimento de programação de ajustes posturais de acordo com a perturbação prevista ${ }^{17}$. Neste caso, conjeturamos que o sistema nervoso central é capaz de dissociar em maior grau a tarefa manual do controle postural ${ }^{41}$, que supostamente é uma estratégia benéfica para se atingir os objetivos de cada componente da tarefa dual.

Como conclusão final, nossos dados mostraram que na tarefa dual aqui avaliada a previsibilidade do tempo de perturbação afetou as respostas manuais e sua integração com as respostas posturais, enquanto que respostas posturais automáticas foram mais estáveis na condição de previsibilidade temporal da perturbação associada à baixa demanda de precisão espacial na tarefa manual. Como comentário adicional, este estudo revela a factibilidade do método empregado para 
avaliar a integração entre controle postural e de tarefas manuais, na busca de melhor compreender a regulação de ações duais dessa natureza realizadas frequentemente em nosso cotidiano. Em estudos futuros, sugerimos que as perturbações sejam aplicadas ao tronco do participante ou por meio de deslocamento de sua base de suporte, evitando-se perturbações diretamente nos braços.

\begin{abstract}
The aim of this study was to assess the integration of the manual and postural components of a dual motor task in response to a perturbation affecting both of those components. Eight healthy young subjects were assessed in a task requiring reactive postural response caused by the release of a load, while pointing a laser continuously to a circular target. The comparisons were performed as a function of the combination of two factors: (a) predictability of the postural perturbation onset, comparing conditions of temporal cueing versus no cueing; and (b) precision demand of the manual task, contrasting targets with diameters of $2 \mathrm{~cm}$ (high constraint) versus $20 \mathrm{~cm}$ (low constraint). Results revealed that temporal predictability of the perturbation led to reduced amplitude of manual movements and to a diminished correlation between manual and postural responses in the condition of temporal predictability associated to low constraint of the manual task. This study reveals the feasibility of the method employed here to evaluate the integration between postural control and manual tasks to better understand the regulation of dual actions performed frequently in our daily lives.
\end{abstract}

\title{
Referências
}

1. Jacobs JV, Horak FB. Cortical control of postural responses. J Neural Transm. 2007;114(10):1339-48.

2. Bardy BG, Marin L, Stoffregen TA, Bootsma RJ. Postural coordination modes considered as emergent phenomena. J Exp Psychol Hum Percept Perform. 1999;25(5):1284-301.

3. Stoffregen TA, Pagulayan RJ, Bardy BB, Hettinger LJ. Modulating postural control to facilitate visual performance. Hum Mov Sci. 2000;19(2):203-20.

4. Berrigan F, Simoneau M, Martin O, Teasdale N. Coordination between posture and movement: interaction between postural and accuracy constraints. Exp Brain Res. 2006;170(2):255-64.

5. Morioka S, Hiyamizu M, Yagi F. The effects of an attentional demand tasks on standing posture control. J Physiol Anthropol Appl Human Sci. 2005;24(3):215-9.

6. Prado JM, Stoffregen TA, Duarte M. Postural sway during dual tasks in young and elderly adults. Gerontology. 2007;53(5):274-81.

7. Stoffregen TA, Bardy BG, Bonnet CT, Hove P, Oullier O. Postural sway and the frequency of horizontal eye movements. Motor Control. 2007;11(1):86-102.

8. Stoffregen TA, Hove P, Bardy BG, Riley M, Bonnet CT. Postural stabilization of perceptual but not cognitive performance. J Mot Behav. 2007;39(2):126-38.

9. Stoffregen TA, Riccio GE. An ecological theory of orientation and the vestibular system. Psychol Rev. 1988;95(1):314.

10. Haddad JM, Ryu JH, Seaman JM, Ponto KC. Time-to-contact measures capture modulations in posture based on the precision demands of a manual task. Gait Posture. 2010;32(4):592-6.

11. Horak FB, Diener HC, Nashner LM. Influence of central set on human postural responses. J Neurophysiol. 1989;62(4):841-53.

12. de Lima AC, de Azevedo Neto RM, Teixeira LA. On the functional integration between postural and supra-postural tasks on the basis of contextual cues and task constraint. Gait Posture. 2010;32(4):615-8.

13. de Lima-Pardini AC, Coelho DB, Silva MB, Azzi NM, Martinelli AR, Horak FB, et al. Aging increases flexibility of postural reactive responses based on constraints imposed by a manual task. Front Aging Neurosci. 2014;6:327.

14. de Lima-Pardini AC, Papegaaij S, Cohen RG, Teixeira LA, Smith BA, Horak FB. The interaction of postural and 
voluntary strategies for stability in Parkinson's disease. J Neurophysiol. 2012;108(5):1244-52.

15. Papegaaij S, de Lima-Pardini AC, Smith BA, Otten E, Cohen RG, Horak FB. Keeping your balance while balancing a cylinder: interaction between postural and voluntary goals. Exp Brain Res. 2012;223(1):79-87.

16. Coelho DB, Bourlinova C, Teixeira LA. Higher order balance control: distinct effects between cognitive task and manual steadiness constraint on automatic postural responses. Hum Mov Sci. 2016;50:62-72.

17. Jacobs JV, Fujiwara K, Tomita H, Furune N, Kunita K, Horak FB. Changes in the activity of the cerebral cortex relate to postural response modification when warned of a perturbation. Clin Neurophysiol. 2008;119(6):1431-42.

18. Marlin A, Mochizuki G, Staines WR, McIlroy WE. Localizing evoked cortical activity associated with balance reactions: does the anterior cingulate play a role? J Neurophysiol. 2014;111(12):2634-43.

19. Mochizuki G, Boe S, Marlin A, McIlRoy WE. Perturbation-evoked cortical activity reflects both the context and consequence of postural instability. Neuroscience. 2010;170(2):599-609.

20. Varghese JP, Marlin A, Beyer KB, Staines WR, Mochizuki G, McIlroy WE. Frequency characteristics of cortical activity associated with perturbations to upright stability. Neurosci Lett. 2014;578:33-8.

21. Fujio K, Obata H, Kawashima N, Nakazawa K. The effects of temporal and spatial predictions on stretch reflexes of ankle flexor and extensor muscles while standing. PLoS One. 2016;11(7):e0158721.

22. Safavynia SA, Ting LH. Long-latency muscle activity reflects continuous, delayed sensorimotor feedback of task-level and not joint-level error. J Neurophysiol. 2013;110(6):1278-90.

23. Welch TD, Ting LH. Mechanisms of motor adaptation in reactive balance control. PLos One. 2014;9(5):e96440.

24. Silva MB, Coelho DB, de Lima-Pardini AC, Martinelli AR, Baptista TS, Ramos RT, et al. Precueing time but not direction of postural perturbation induces early muscular activation: comparison between young and elderly individuals. Neurosci Lett. 2015;588:190-5.

25. Coelho DB, Teixeira LA. Cognition and balance control: does processing of explicit contextual cues of impending perturbations modulate automatic postural responses? Exp Brain Res. 2017;235(8):2375-90.

26. Mochizuki G, Sibley KM, Cheung HJ, McIlroy WE. Cortical activity prior to predictable postural instability: Is there a difference between self-initiated and externally-initiated perturbations? Brain Res. 2009;1279:29-36.

27. Mihara M, Miyai I, Hatakenaka M, Kubota K, Sakoda S. Role of the prefrontal cortex in human balance control. Neuroimage. 2008;43(2):329-36.

28. Andersen RA, Snyder LH, Bradley DC, Xing J. Multimodal representation of space in the posterior parietal cortex and its use in planning movements. Annu Rev Neurosci. 1997;20:303-30.

29. Slobounov S, Wu T, Hallett M. Neural basis subserving the detection of postural instability: an fMRI study. Motor Control. 2006;10(1):69-89.

30. Pellijeff A, Bonilha L, Morgan PS, McKenzie K, Jackson SR. Parietal updating of limb posture: an event-related fMRI study. Neuropsychologia. 2006;44(13):2685-90.

31. Ferraye MU, Debû B, Heil L, Carpenter M, Bloem BR, Toni I. Using motor imagery to study the neural substrates of dynamic balance. PLoS One. 2014;9(3):e91183.

32. Fujimoto H, Mihara M, Hattori N, Hatakenaka M, Kawano T, Yagura H, et al. Cortical changes underlying balance recovery in patients with hemiplegic stroke. Neuroimage. 2014;85:547-54.

33. Mierau A, Hulsdunker T, Struder HK. Changes in cortical activity associated with adaptive behavior during repeated balance perturbation of unpredictable timing. Front Behav Neurosci. 2015;9:1-12.

34. Haddad JM, Rietdyk S, Claxton LJ, Huber JE. Task-dependent postural control throughout the lifespan. Exerc Sport Sci Rev. 2013;41(2):123-32.

35. Rankin JK, Woollacott MH, Shumway-Cook A, Brown LA. Cognitive influence on postural stability: a neuromuscular analysis in young and older adults. J Gerontol A Biol Sci Med Sci. 2000;55(3):M112-9.

36. Lajoie Y, Teasdale N, Bard C, Fleury M. Attentional demands for static and dynamic equilibrium. Exp Brain Res. 1993;97(1):139-44.

37. Lajoie Y, Teasdale N, Bard C, Fleury M. Upright standing and gait: are there changes in attentional requirements related to normal aging? Exp Aging Res. 1996;22(2):185-98.

38. Maylor EA, Wing AM. Age differences in postural stability are increased by additional cognitive demands. J Gerontol B Psychol Sci Soc Sci. 1996;51(3):P143-54.

39. Little CE, Woollacott M. Effect of attentional interference on balance recovery in older adults. Exp Brain Res. 2014;232(7):2049-60.

40. Little CE, Woollacott M. EEG measures reveal dual-task interference in postural performance in young adults. Exp 
Silva AG, et al.

Brain Res. 2015;233(1):27-37.

41. Amado AC, Palmer CJ, Hamill J, van Emmerik RE. Coupling of postural and manual tasks in expert performers. Hum Mov Sci. 2016;46:251-60.

42. Tagliabue M, Ferrigno G, Horak F. Effects of Parkinson's disease on proprioceptive control of posture and reaching while standing. Neuroscience. 2009;158(4):1206-14.

43. Pozzo T, Stapley PJ, Papaxanthis C. Coordination between equilibrium and hand trajectories during whole body pointing movements. Exp Brain Res. 2002;144(3):343-50.

ENDEREÇO DE CORRESPONDÊNCIA:

Daniel Boari Coelho

Av. Prof. Mello Moraes, 65 - Butantã

São Paulo - SP - BRASIL

CEP: 05508-030

E-mail: daniboari@usp.br
Submetido: 23/11/2016

$1^{\mathrm{a}}$ revisão: 04/04/2017

$2^{\mathrm{a}}$ revisão: 15/o8/2017

Aceito: 16/08/2017 